\title{
On the Substructure of the Cosmological Constant
}

\author{
Gia Dvali, ${ }^{a b c}$ César Gómez ${ }^{d}$ and Sebastian Zell ${ }^{* a b}$ \\ ${ }^{a}$ Arnold Sommerfeld Center, Ludwig-Maximilians-Universität, \\ Theresienstraße 37, 80333 München, Germany \\ ${ }^{b}$ Max-Planck-Institut für Physik, \\ Föhringer Ring 6, 80805 München, Germany \\ ${ }^{c}$ Center for Cosmology and Particle Physics, Department of Physics, New York University, \\ 4 Washington Place, New York, NY 10003, USA \\ ${ }^{d}$ Instituto de Física Teórica UAM-CSIC, Universidad Autónoma de Madrid, \\ Cantoblanco, 28049 Madrid, Spain \\ E-mail: sebastian.zell@campus. $1 \mathrm{mu}$.de
}

We summarize the findings of our paper [1]. We start by defining the quantum break-time. Once one understands a classical solution as expectation value of an underlying quantum state, it emerges as time-scale after which the true quantum evolution departs from the classical mean field evolution. We apply this idea to de Sitter space. Following [2], we construct a simple model of a spin-2 field, which for some time reproduces the de Sitter metric and simultaneously allows for its well-defined representation as coherent quantum state of gravitons. The mean occupation number $N$ of background gravitons turns out to be equal to the de Sitter horizon area in Planck units, while their frequency is given by the de Sitter Hubble parameter. In the semi-classical limit, we show that the model reproduces all semi-classical calculations in de Sitter, such as thermal Gibbons-Hawking radiation, all in the language of quantum $S$-matrix scatterings and decays of coherent state gravitons. Most importantly, this framework allows to capture the $1 / N$-effects of back reaction to which the usual semi-classical treatment is blind. They violate the de Sitter symmetry and lead to a finite quantum break-time of the de Sitter state equal to the de Sitter radius times $N$. We also point out that the quantum-break time is inversely proportional to the number of particle species in the theory. Thus, the quantum break-time imposes the following consistency condition: Older and species-richer universes must have smaller cosmological constants. For the maximal, phenomenologically acceptable number of species, the observed cosmological constant would saturate this bound if our Universe were $10^{100}$ years old in its entire classical history.

Corfu Summer Institute 2016 "School and Workshops on Elementary Particle Physics and Gravity" 31 August - 23 September, 2016

Corfu, Greece

* Speaker. 


\section{The world is fundamentally quantum}

At the fundamental level, nature is quantum, i.e., Planck's constant $\hbar$ is finite. Thus, any classical solution must be seen as approximation of an underlying quantum dynamic. Despite that fact, the classical mean field description works very well in many cases. Namely, this is true for systems with large occupation number of quanta, $N \gg 1$. They behave more and more classically with $N \rightarrow \infty$. For example, in quantum electrodynamics a coherent state of photons of a given frequency and a large mean occupation number is well described by a classical electromagnetic wave. This description is excellent up to observations which are sensitive to individual photons. Such effects correct the evolution of the system by $1 / N$ relative to the leading order classical evolution.

If the system is interacting, however, there exist quantum processes to which this mean field description is blind. Although these processes, which take into account rescatterings of the individual quanta, are suppressed, they are important because they lead to departures of the true quantum evolution of the system from the classical description and cause a complete breakdown of the latter after some time. Following [3], we shall refer to this time-scale as quantum break-time $t_{\mathrm{q}}$. The above argument implies that essentially any classical system has an associated quantum break-time.

In the following, we shall apply this concept to the classical de Sitter solution of gravity, thereby summarizing the results of our paper [1]. As a first step, we implement in section 2 the earlier approach of [2] to construct a simple but explicit model which provides a quantum description of de Sitter. The fundamental quanta of this model are defined as spin-2 excitations on a Minkowski vacuum. We use those to construct a coherent state of high graviton occupancy and show that during the regime of validity of our model, its expectation value is equal to a classical de Sitter metric.

Subsequently, we demonstrate in section 3 that the semi-classical approximation corresponds to taking the limit $N \rightarrow \infty$ in which any back reaction to the classical mean field can be neglected. In this limit, we fully reproduce the the semi-classical evolution of probe particles in the fixed classical metric.

In section 4, we proceed to study particle production in a de Sitter universe. In our quantum description, we can compute it as scattering process of the constituent gravitons. Using this example of particle production, we point out the crucial novelty of our quantum picture: It allows us to identify processes to which the standard semi-classical treatment is blind. These processes lead to departures from the classical evolution and therefore to a quantum break-time of the classical mean field description. They are suppressed by $1 / N$, but are cumulative in nature and start to dominate over a sufficiently long time-scale. In particular, this implies that the description of de Sitter in terms of a classical metric cannot be future-eternal. We estimate the quantum break-time as $t_{\mathrm{q}} \sim M_{p}^{2} / \Lambda^{3 / 2}[2,4]$, where $M_{p}$ is the Planck mass and $\Lambda$ the cosmological constant.

Finally, we will discuss in section 5 that our framework turns the cosmological constant problem from an issue of naturalness into a question of quantum-mechanical consistency [4]. We explicitly estimate the maximal duration of the entire classical history of our Universe as well as the maximal value of the cosmological constant consistent with the age of our Universe.

We conclude by summarizing our findings in section 6 . 


\section{A quantum description of the de Sitter metric}

\subsection{Approximation on the classical level}

We start from the de Sitter metric in closed Friedmann-Robertson-Walker slicing:

$$
\mathrm{d} s^{2}=\mathrm{d} t^{2}-\cosh ^{2}(\sqrt{\Lambda / 3} t)\left(\frac{\mathrm{d} r^{2}}{1-\Lambda r^{2} / 3}+r^{2} \mathrm{~d} \Omega^{2}\right) .
$$

Our first goal is to find a quantum description of this metric. For the full nonlinear classical solution, however, it is impossible to do so. To overcome this problem, our strategy is to approximate the classical solution so that an exact quantum description can be found for the modified classical solution. It is crucial to note that this approximation only happens on the classical level. When the quantum description of the classical solution breaks down, this only happens because the approximation of the exact solution on the classical level stops being valid. We define the time-scale after which the exact and approximate classical solution deviate significantly as classical breaktime $t_{\mathrm{cl}}$. We point out that this quantity is not fundamental but only a consequence of the particular approximation we choose.

In order to find a suitable approximation, we follow [5], where Pauli-Fierz theory of a massive spin-2 was studied. There, it was shown that the free theory with mass $m=\sqrt{\Lambda}$ can reproduce the massless solution (2.1) for short times $t \ll \Lambda^{-1 / 2}$. On this time-scale, the graviton mass therefore has the same effect as the cosmological constant term. This means that observers coupled to such a gravitational field, for a short time-scale cannot tell whether they live in a de Sitter metric of Einstein theory or in a coherently oscillating field of a massive Fierz-Pauli graviton on top of a flat Minkowski vacuum. We will first recapitulate this result and then discuss its physical meaning.

We proceed to modify our theory by adding a graviton mass $m$ and removing the cosmological constant source. For short time-scales $t \ll m^{-1}=\Lambda^{-1 / 2}$, it is moreover possible to linearize the theory on top of the Minkowski metric $\eta_{\mu \nu}$. In this case, the massive graviton, which we shall denote as $h_{\mu v}$, obeys:

$$
\varepsilon_{\mu \nu}^{\alpha \beta} h_{\alpha \beta}+m^{2}\left(h_{\mu \nu}-\eta_{\mu \nu} h\right)=0,
$$

with the linearized Einstein tensor defined as $\varepsilon_{\mu \nu}^{\alpha \beta} h_{\alpha \beta} \equiv \square h_{\mu v}-\eta_{\mu v} \square h-\partial_{\mu} \partial^{\alpha} h_{\alpha v}-\partial_{v} \partial^{\alpha} h_{\alpha \mu}+$ $\partial_{\mu} \partial_{v} h+\eta_{\mu v} \partial^{\alpha} \partial^{\beta} h_{\alpha \beta}$. It is very important not to confuse $h_{\mu v}$ with the linear metric perturbation on top of a de Sitter metric: As we shall see, $h_{\mu v}$ is a short-time approximation of the de Sitter metric itself, not a fluctuation on top of it. In addition to (2.2), the massive graviton must satisfy the Fierz-Pauli constraint

$$
\partial^{\mu}\left(h_{\mu v}-\eta_{\mu v} h\right)=0
$$

which shows that it propagates five degrees of freedom. Following $[5,6]$, these degrees of freedom can be split according to irreducible massless representations of the Poincaré group into three different helicity components: helicity- $2 \widetilde{h}_{\mu \nu}$, helicity- $1 A_{\mu}$ and helicity- $0 \chi$ :

$$
h_{\mu v}=\widetilde{h}_{\mu v}+\frac{1}{m}\left(\partial_{\mu} A_{v}+\partial_{v} A_{\mu}\right)+\frac{1}{6} \eta_{\mu v} \chi-\frac{1}{3} \frac{\partial_{\mu} \partial_{v}}{m^{2}} \chi .
$$


This decomposition is unique in the sense that in this basis, the kinetic mixing among different helicities is absent and in the limit $m \rightarrow 0$, the field $h_{\mu v}$ "disintegrates" into three independent massless representations of the Poincaré group: spin-2, spin-1 and spin-0. Following [6], we can integrate out the additional helicities and write down an effective equation for $\widetilde{h}_{\mu v}$. This equation in de Donder gauge, $\partial^{\mu} \widetilde{h}_{\mu v}=\frac{1}{2} \partial_{v} \widetilde{h}$, is a massive wave equation:

$$
\left(\square+m^{2}\right)\left(\widetilde{h}_{\mu v}-\frac{1}{2} \eta_{\mu v} \widetilde{h}\right)=0 .
$$

One solution is given by

$$
\begin{aligned}
\widetilde{h}_{00} & =-\frac{2 \Lambda}{m^{2}} \cos (m t) \\
\widetilde{h}_{0 i} & =\frac{-2 \Lambda}{3 m} \sin (m t) x_{i} \\
\widetilde{h}_{i j} & =\frac{2 \Lambda}{m^{2}} \cos (m t) \delta_{i j}-\frac{\Lambda}{3} \cos (m t) \varepsilon_{i j}
\end{aligned}
$$

If we set $m=\sqrt{\Lambda}$, it is easy to check that this oscillating solution of the massive theory without any cosmological constant indeed reproduces - up to an additive constant - the de Sitter solution (2.1) of the massless theory with a cosmological constant as source. Obviously, the classical break-time after which this approximation breaks down is

$$
t_{\mathrm{cl}}=\Lambda^{-1 / 2}
$$

But why is the approximation (2.6) in terms of massive gravity useful? On a computational level, its crucial feature is that - unlike the massless solution which possesses a source - it is the solution of a free theory. Therefore, it can be quantized in a straightforward way and allows for a simple coherent state interpretation of the de Sitter metric.

In addition, it matches the physical intuition that if de Sitter space allows for a sensible corpuscular resolution in form of a coherent state, the constituents must have frequencies given by the Hubble parameter since this is the only scale of the classical geometry. Thus, these constituents can be viewed as some sort of off-shell gravitons of non-zero frequencies set by $H$. The mass term is the simplest terms which provides such an effective off-shell dispersion relation. Thus, for a sufficiently short time-interval, $t \ll t_{\mathrm{cl}}$, we can think of the gravitons of the massless theory which are put off-shell by nonlinearities as on-shell massive gravitons of a free theory. This mapping allows for the coherent state interpretation of de Sitter metric for sufficiently small times. Although the approximation breaks down after $t_{\mathrm{cl}}$, it suffices to "fish out" the $1 / N$-quantum effects which lead to a departure from the coherent state picture.

By no means, however, should one think that the cosmological term gives the graviton a fundamental mass. This is not the case as it is obvious already from counting the number of degrees of freedom. We only use the fact that the Einsteinian spin-2 helicity component $\tilde{h}_{\mu v}$ of the FierzPauli massive graviton without cosmological term has the same form as it would have in a massless theory with cosmological constant. 


\subsection{The fundamental Lagrangian}

So far, we have merely approximated the classical solution. Our next step is to find a quantum description for the metric (2.6). Since we work in the linear theory of Pauli-Fierz massive gravity, our effective Lagrangian on the quantum level is

$$
\hat{\mathscr{L}}_{\mathrm{FP}}=\frac{1}{16 \pi}\left(\frac{1}{2} \hat{h}^{\mu v} \varepsilon_{\mu v}^{\alpha \beta} \hat{h}_{\alpha \beta}+\frac{1}{2} \Lambda\left(\hat{h}_{\mu v} \hat{h}^{\mu v}-\hat{h}^{2}\right)+16 \pi \frac{1}{M_{p}} \hat{\tilde{h}}_{\mu v} \hat{T}^{\mu v}(\hat{\Psi})+\ldots\right) .
$$

It is crucial to note that it does not include a cosmological constant-term. Instead, it only contains the Fierz-Pauli mass term $\left(\hat{h}_{\mu v} \hat{h}^{\mu v}-\hat{h}^{2}\right)$. We moved to canonically normalized classical fields by multiplying with the Planck mass $M_{p}$. The helicity decomposition of $\hat{h}_{\mu v}$ continues to have the form given by (2.4).

$\hat{T}^{\mu v}(\Psi)$ denotes the energy momentum tensor of all modes $\hat{\Psi}$ which do not belong to the mode-decomposition of the background de Sitter solution, i.e., the quanta which in the quantum picture are not part of the coherent state description of the de Sitter metric. We only keep the lowest order dependence of $\hat{T}^{\mu v}(\hat{\Psi})$ on the fields. Notice that we couple $\hat{T}^{\mu v}(\hat{\Psi})$ only to the Einstein spin2 helicity component $\hat{\tilde{h}}_{\mu v}$. The reason why we do not couple the $\hat{\chi}$-component to external sources is that we want the external particles $\hat{\Psi}$ to experience - in the classical limit and during the timescale (2.7) of validity of our model - "life" in an effective de Sitter metric. ${ }^{1}$ As explained above, the classical solution (2.6) for the helicity-2 part $\widetilde{h}_{\mu v}$ alone suffices for that since it reproduces the de Sitter solution (2.1) of the massless theory for $t<t_{\mathrm{cl}}$.

For definiteness, we finally assume that $\hat{T}^{\mu \nu}$ is the stress-energy tensor of some external particles and does not contain a graviton part. Including $\hat{h}_{\mu \nu}$ into $\hat{T}^{\mu \nu}$ would result in nonlinear self-interactions of $\hat{h}_{\mu v}$, which would contribute to both classical and quantum break-times. Since our approach is to replace the effect of the self-coupling by an effective graviton mass, we will not include gravitons in $\hat{T}^{\mu v}(\hat{\Psi})$. In addition to that, it is convenient to couple gravity exclusively to external particles with initial occupation number equal to zero because such particles manifest themselves only via quantum processes and this is precisely what we are after.

Finally, we further split the helicity-2 component $\hat{\breve{h}}_{\mu v}$ according to the symmetries of the Poincaré group. It suffices for our conclusions to focus exclusively on the scalar part, i.e., the trace of helicity-2 component $\hat{\widehat{h}}_{\mu \nu}$ :

$$
\hat{\tilde{h}}_{\mu v}^{\mathrm{s}}=-\sqrt{16 \pi} \hat{\Phi} \eta_{\mu v}
$$

which we can effectively describe as massive scalar field: ${ }^{2}$

$$
\hat{\mathscr{L}}=\frac{1}{2}\left(\left(\partial_{\mu} \hat{\Phi}\right)^{2}-\Lambda \hat{\Phi}^{2}\right)+\frac{-\sqrt{16 \pi}}{M_{P}} \hat{\Phi}_{\mu}^{\mu}(\hat{\Psi}) .
$$

\subsection{The quantum state}

According to (2.6), the scalar component has the expectation value

$$
\Phi=v \cos (\sqrt{\Lambda} t)
$$

\footnotetext{
${ }^{1}$ Notice that the coupling of the helicity-1 component $\hat{A}_{\mu}$ to $\hat{T}_{\mu v}$ automatically vanishes due to the conservation of the source: $\partial^{\mu} \hat{T}_{\mu v}=0$.

${ }^{2}$ Note that the Pauli-Fierz constraint (2.3) in de Donder gauge implies that $\hat{\chi}=-\hat{\tilde{h}}$. Thus, $\hat{\Phi}$ also describes $\hat{\chi}$.
} 
with $v:=\frac{M_{p}}{\sqrt{4 \pi}}$. Thus, our final task consists in choosing a quantum state $|N\rangle$ of the field $\hat{\Phi}$ such that $\langle N|\hat{\Phi}| N\rangle=v \cos (\sqrt{\Lambda} t)$. We start by expanding $\hat{\Phi}$ in creation and annihilation operators:

$$
\hat{\Phi}=\int \frac{\mathrm{d}^{3} \vec{k}}{\sqrt{(2 \pi)^{3} 2 \sqrt{\Lambda+\vec{k}^{2}}}}\left(\hat{a}_{\vec{k}} e^{-i k x}+\hat{a}_{\vec{k}}^{\dagger} e^{i k x}\right),
$$

with the standard commutation relations $\left[\hat{a}_{\vec{k}}, \hat{a}_{\vec{k}^{\prime}}^{\dagger}\right]=\delta^{(3)}\left(\vec{k}-\vec{k}^{\prime}\right)$.

If we do not consider external fields in (2.10), $\hat{\Phi}$ is a free field, i.e., (2.12) gives the full Heisenberg operator. As explained before, it is crucial for the validity of our approach that we consider a free field since only in that case, we can use the Fock space of the free operators $\hat{a}_{\vec{k}}, \hat{a}_{\vec{k}}^{\dagger}$ to construct the quantum state $|N\rangle$. In the presence of interaction, we would have to use different operators with a modified dispersion relation. Those are, however, generically impossible to define. It is the approximation (2.6) of the classical solution which enables us to use a free field. The selfconsistency of using this free massive scalar field justifies our choice of classical approximation.

Since we want $|N\rangle$ to reproduce the classical expectation value (2.11), we choose it maximally classical, i.e, as coherent state. As the expectation value is furthermore translation-invariant, we only need to consider quanta of zero momentum. Thus, we use the ansatz

$$
\begin{aligned}
|N\rangle & =\mathrm{e}^{-\frac{1}{2} N} \sum_{n=0}^{\infty} \frac{N^{\frac{n}{2}}}{\sqrt{n !}}|n\rangle, \text { with } \\
N & =\frac{V \sqrt{\Lambda} M_{p}^{2}}{8 \pi} .
\end{aligned}
$$

In this formula, $|n\rangle=\left(\left((2 \pi)^{3} / V\right)^{1 / 2} \hat{a}_{\overrightarrow{0}}^{\dagger}\right)^{n}(n !)^{-\frac{1}{2}}|0\rangle$ are normalized number eigenstates of $n$ quanta with zero momentum. Using the fact that coherent states are eigenvectors of the annihilation operator, we conclude that the state $|N\rangle$ indeed yields the correct expectation value:

$$
\langle N|\hat{\Phi}| N\rangle=\frac{1}{\sqrt{2 \sqrt{\Lambda}}}\left(\sqrt{\frac{N}{V}} \mathrm{e}^{-i \sqrt{\Lambda} t}+\sqrt{\frac{N}{V}} \mathrm{e}^{i \sqrt{\Lambda} t}\right)=v \cos (\sqrt{\Lambda} t),
$$

where we plugged in the mode expansion (2.12). Thus, we have achieved our goal. We found a quantum description of the classical metric (2.9) in terms of the Lagrangian (2.10) and the state (2.13). In one Hubble volume, $V \propto \Lambda^{-3 / 2}$, we get

$$
N_{\mathrm{H}}=\frac{M_{p}^{2}}{\Lambda}
$$

gravitons, in accordance with [2].

We conclude by calculating the expectation value of the energy of the coherent state (2.13). It is given by the product of the graviton rest mass and their average occupation number in the coherent state:

$$
E_{\text {quant }}=\sqrt{\Lambda} N
$$

If we plug in (2.14), we conclude that this is indeed equal to the classical energy associated to the cosmological constant: $E_{\text {class }}=\frac{\Lambda M_{p}^{2} V}{8 \pi}$. That our quantum description reproduces the correct classical energy is a consistency check for our approach. 


\section{Uncovering the quantum origin of classical evolution}

\subsection{The semi-classical limit}

Our goal so far was to find a quantum description of the de Sitter metric in terms of the expectation value of the coherent state (2.13). The first evidence which supports the validity of such a modeling is that the state of free massive gravitons reproduces the correct classical expectation value. Of course, this argument does not suffice, since one can realize a given expectation value in a multitude of ways. Therefore, we will collect further evidence by demonstrating that our framework is constructed such that it automatically reproduces all semi-classical results in de Sitter.

Our starting point is the Lagrangian (2.10):

$$
\hat{\mathscr{L}}=\frac{1}{2}\left(\left(\partial_{\mu} \hat{\Phi}\right)^{2}-m^{2} \hat{\Phi}^{2}+\left(\partial_{\mu} \hat{\Psi}\right)^{2}-m_{\Psi}^{2} \hat{\Psi}^{2}\right)+\frac{\hat{\Phi}}{M_{P}} \hat{T}_{\mu}^{\mu}(\hat{\Psi}),
$$

where we use a massive scalar $\hat{\Psi}$ as external field for simplicity. Its stress-energy tensor is $\hat{T}^{\mu v}(\hat{\Psi}) \equiv$ $-\sqrt{16 \pi}\left(\partial^{\mu} \hat{\Psi} \partial^{\nu} \hat{\Psi}-\frac{1}{2} \partial^{\alpha} \hat{\Psi} \partial_{\alpha} \hat{\Psi} \eta^{\mu \nu}+\frac{1}{2} m_{\Psi}^{2} \hat{\Psi}^{2} \eta^{\mu \nu}\right)$. In order to simplify notations, we chose the unconventional normalization $-\sqrt{16 \pi}$ to account for the corresponding factor in (2.10).

In general, the semi-classical treatment corresponds to quantizing weak field excitations on top of a fixed classical background metric, i.e., ignoring any back reaction to the metric from the creation and propagation of quantum particles. In our model, this amounts to quantizing the $\hat{\Psi}$ field in an effective de Sitter space-time created by the classical $\Phi$-field. Thus, we can derive the standard semi-classical evolution of a probe particle $\hat{\Psi}$ in the background classical metric from the effective Lagrangian

$$
\hat{\mathscr{L}}_{\hat{\Psi}}^{(\mathrm{eff})}=\frac{1}{2}\left(\left(\partial_{\mu} \hat{\Psi}\right)^{2}-m_{\Psi}^{2} \hat{\Psi}^{2}\right)+\frac{\Phi_{\mathrm{cl}}}{M_{P}} \hat{T}_{\mu}^{\mu}(\hat{\Psi}),
$$

which can be obtained from (3.1) when we replace the quantum field $\hat{\Phi}$ by the classical solution, $\hat{\Phi} \rightarrow \Phi_{\mathrm{cl}}$. Here $\Phi_{\mathrm{cl}}$ is given by (2.11). In such a treatment, the only relevant asymptotic quantum states are the initial states $\left|i_{\Psi}\right\rangle$ and the final states $\left|f_{\Psi}\right\rangle$ of the $\hat{\Psi}$-field since the background metric is a $c$-number.

The fact that we are treating $\Phi_{\mathrm{cl}}$ as a fixed classical background means that we are taking the limit:

$$
\Lambda=m^{2}=\text { fixed, } M_{P} \rightarrow \infty .
$$

In that case, any back reaction from the dynamics of $\hat{\Psi}$ can be ignored and we can treat $\Phi_{\mathrm{cl}}$ as an eternal classical background. In the quantum picture, this corresponds to taking the limit

$$
\Lambda=m^{2}=\text { fixed }, N \rightarrow \infty,
$$

i.e., to using a coherent state with infinite mean occupation number. It is important to note that we keep $\hbar$ fixed in both limits. For convenience, we have set $\hbar=1$.

\subsection{The fully quantum picture}

As we have seen, the replacement $\hat{\Phi} \rightarrow \Phi_{\mathrm{cl}}$ suffices to obtain the semi-classical limit. However, we are asking for more. In our theory, this approximation must emerge as a result of fully quantum 
interactions between the metric-quanta and external particles $\hat{\Psi}$. This means that we would like to understand this replacement not as an external prescription, but as a result of taking the limit (3.4) in the full quantum evolution.

In order to achieve this, let us fist describe the evolution of a $\hat{\Psi}$-field in the effective semiclassical theory (3.2) in the language of an $S$-matrix evolution operator. The non-trivial quantum evolution is due to the last term, which represents the off-diagonal part of the Hamiltonian density:

$$
\hat{\mathscr{H}}_{\mathrm{int}}^{(\mathrm{eff})}(x)=\frac{\Phi_{\mathrm{cl}}}{M_{P}} \hat{T}_{\mu}^{\mu}(\hat{\Psi}) .
$$

We can derive the quantum evolution in a weak-field perturbation theory in the expansion parameter $\Phi_{\mathrm{cl}} / M_{P} \ll 1$. To first order in this expansion, we define the non-trivial part of the effective $S$-matrix evolution operator

$$
\hat{S}^{(\text {eff })}=-i \int \mathrm{d}^{4} x \mathbf{T}\left\{\hat{\mathscr{H}}_{\text {int }}^{(\text {eff })}(x)\right\} .
$$

The quantum evolution of $\hat{\Psi}$ is then described by the matrix elements between different initial and final states:

$$
\mathscr{A}=\left\langle\mathrm{f}_{\Psi}\left|\hat{S}^{(\mathrm{eff})}\right| \mathrm{i} \Psi\right\rangle
$$

Of course, since the effective Hamiltonian is explicitly time-dependent, the evolution described by the effective $S$-matrix is in general non-unitary. This leads to subtleties in defining the relevant initial and final $S$-matrix states on such a time-dependent background. This complication is completely standard and is a consequence of taking the zero back reaction limit.

Our immediate goal is not to enter in these well-known issues, but rather to understand the effective semi-classical evolution as the limit of the underlying fully quantum one. For this it is enough to recall that the coherent state $|N\rangle$ is defined in such a way that it reproduces the classical metric in form of the expectation value:

$$
\Phi_{\mathrm{cl}}=\langle N|\hat{\Phi}| N\rangle
$$

Consequently, the effective semi-classical $S$-matrix operator can be written as

$$
\left\langle\mathrm{f}_{\Psi}\left|\hat{S}^{(\mathrm{eff})}\right| \mathrm{i}_{\Psi}\right\rangle=\left(\left\langle\mathrm{f}_{\Psi}|\otimes\langle N|) \hat{S}\left(|N\rangle \otimes\left|\mathrm{i}_{\Psi}\right\rangle\right),\right.\right.
$$

where $\hat{S}$ is the full quantum $S$-matrix evolution operator ${ }^{3}$

$$
\hat{S}=-i \int \mathrm{d}^{4} x \mathbf{T}\left\{\hat{\mathscr{H}}_{\text {int }}(x)\right\}
$$

defined by the full quantum interaction Hamiltonian

$$
\hat{\mathscr{H}}_{\text {int }}(x)=\frac{\hat{\Phi}}{M_{P}} \hat{T}_{\mu}^{\mu}(\hat{\Psi}) .
$$

\footnotetext{
${ }^{3}$ It will turn out that the coherent state $|N\rangle$ evolves in time. Therefore, the $S$-matrix calculation is only applicable when the time-scale of the process which we study is much shorter than the one on which $|N\rangle$ changes. As we will show in section 4.3 , this is indeed the case.
} 
This means that the initial state in the fully quantum picture does not only consist of the external particles $\left|i_{\Psi}\right\rangle$. Instead, we use the coherent state (describing de Sitter) with external particles on top of it: $|N\rangle \otimes\left|i_{\Psi}\right\rangle$. Likewise, the final state is $|N\rangle \otimes\left|f_{\Psi}\right\rangle$.

Of course, the true quantum evolution inevitably implies transitions to final graviton states $\left|N^{\prime}\right\rangle$ which differ from the initial coherent state $|N\rangle$ and in general are not even coherent. Such transitions are not equivalent to simply replacing the graviton field by its expectation value and lead to departures from semi-classicality. Therefore, equation (3.9) makes the quantum meaning of the semi-classical limit apparent: It corresponds to setting $\left|N^{\prime}\right\rangle=|N\rangle$, i.e., ignoring any back reaction to the graviton state. Thus, the semi-classical $S$-matrix elements, which reproduce the motion of an external $\hat{\Psi}$-particle in the classical metric, are the subset of fully quantum $S$-matrix elements in which the quantum field $\hat{\Phi}$ is taken in the same initial and final state $|N\rangle$.

Notice that this selection of matrix elements is automatic in the limit (3.4), due to standard properties of coherent states. This is true since the overlap of coherent states scales as

$$
\langle N+\Delta N \mid N\rangle=\exp \left(\frac{-\Delta N^{2}}{8 N}\right)
$$

so that $\langle N+\Delta N \mid N\rangle \approx\langle N \mid N\rangle=1$ for $N \rightarrow \infty$. In that case, we can set $\left|N^{\prime}\right\rangle \approx|N\rangle$. This consistently explains why this limit corresponds to a zero back reaction. A similar argument holds for transitions from the initial coherent state to non-coherent ones.

For explicit examples on how our quantum description of de Sitter reproduces semi-classical results we refer the reader to [1], where, among others, we discuss the process of redshift in de Sitter.

\section{Gibbons-Hawking particle production as quantum decay of the coherent de Sitter state}

\subsection{The fully quantum picture}

It has been a long-standing prediction by Gibbons and Hawking that particle production occurs in a de Sitter background [7]: An observer should see a thermal spectrum of temperature $T \sim \sqrt{\Lambda}$. In their semi-classical treatment, in which quantum fields are studied on top of the undisturbed classical metric background, particle production arises as a vacuum process: Since the vacua of quantum fields depend on time in the evolving de Sitter metric, their early-time vacuum contains particles from the point of view of a late-time observer. As already explained, this semi-classical treatment does not contain a back reaction, i.e., particle production does not change the classical de Sitter metric.

Subsequently, we will study particle production in our fully quantum framework. In doing so, our first goal is to understand how this semi-classical process arises in the language of underlying fully quantum dynamics, thereby making the general argument of the previous section explicit. As we will calculate shortly, the self-coupling of gravitons as well as their coupling to other particle species lead to processes of quantum scattering and decay of the constituent gravitons of de Sitter. The final products of such decays and scatterings contain particles which no longer belong to the coherent state and have dispersion relations of free quanta propagating on a classical de Sitter 


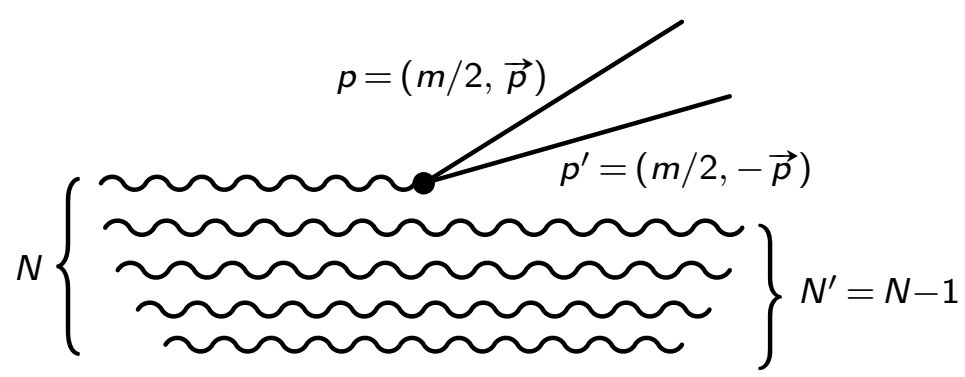

Figure 1: Particle production as graviton decay: One of the $N$ initial gravitons decays and produces 2 external particles of 4-momentum $p$ and $p^{\prime}$.

background. In the usual semi-classical treatment, these processes correspond to the production of Gibbons-Hawking quanta. In our quantum picture, Gibbons-Hawking radiation therefore arises from the decay of coherent state gravitons.

Our main goal, however, is not merely to reproduce the known semi-classical results but to identify new corrections originating from this quantum dynamics which any semi-classical treatment is unable to capture. It will become clear shortly that those effects originate from taking into account the back reaction to the coherent de Sitter state and manifest themselves as $1 / \mathrm{N}$ corrections.

We shall proceed to make more quantitative estimates. For illustrating the point, we limit ourselves to studying the decay processes of background gravitons into external particle species. Due to the universality of graviton coupling, the process of graviton production has a similar rate. For definiteness, we will take as external particles a massive scalar field $\hat{\Psi}$, as in (3.1). Then the leading order contribution to particle production is given by a transition from the initial state $|N\rangle$ to a final state which contains two $\hat{\Psi}$-particles. It is depicted in figure 1 .

Denoting the annihilation operators of the external field $\hat{\Psi}$ by $\hat{b}_{\vec{p}}$, we get the $S$-matrix amplitude of the decay:

$$
\mathscr{A}=\left\langle N^{\prime}\left|\hat{b}_{\vec{p}} \hat{b}_{\vec{p}^{\prime}} \hat{S}\right| N\right\rangle=\mathscr{K}\left(-p, p^{\prime}\right) \int \mathrm{d}^{4} x e^{i\left(p+p^{\prime}\right) x}\left\langle N^{\prime}|\hat{\Phi}| N\right\rangle,
$$

where we plugged in the full $S$-matrix operator (3.10) and $p$ and $p^{\prime}$ are the 4-momenta of the produced $\hat{\Psi}$-particles. In this expression, the gravitational field solely appears in $\left\langle N^{\prime}|\hat{\Phi}| N\right\rangle$ and the kinematical factor $\mathscr{K}\left(p, p^{\prime}\right)$, which only depends on the external particles, reads

$$
\mathscr{K}\left(p, p^{\prime}\right)=i \frac{\sqrt{16 \pi}}{2(2 \pi)^{3} M_{p} \sqrt{p_{0} p_{0}^{\prime}}}\left(p \cdot p^{\prime}-2 m_{\Psi}^{2}\right) .
$$

In comparison to the semi-classical treatment, the crucial novelty of our approach is that it uncovers the existence of quantum processes in which the final state $\left|N^{\prime}\right\rangle$ of background gravitons is different from the initial one $|N\rangle$. These processes correspond to $1 / N$-corrections and therefore are fundamentally invisible in the semi-classical picture which, as explained above, in our framework is reproduced in $N \rightarrow \infty$ limit. In particular, the final state $\left|N^{\prime}\right\rangle$ obtained as a result of particle production in quantum theory does not even have to be a coherent state. This deviation from 
coherence gives a gradual departure from classicality and eventually leads to a quantum breaktime.

However, for the case of de Sitter even the transitions among coherent states with different occupation numbers are sufficient for capturing the departure from the classical evolution since classically de Sitter is an eternal state and simply cannot change. Any quantum process which changes the characteristics of de Sitter space marks a fundamentally new phenomenon not visible in the semi-classical theory. Therefore, for illustrating this point we shall consider transitions to a coherent state, but with different mean occupation number $N^{\prime} \neq N$.

Finally, we need to determine the expectation value of the particle number for the final coherent state. In doing so, we want to disentangle two effects: Since a coherent state is not a particle number eigenstate, its particle number fluctuates even without any interaction, i.e., $\left\langle N^{\prime} \mid N\right\rangle \neq 0$. Our goal is not to consider this non-Hamiltonian effect. Instead, we want to focus on how the coherent state evolves due to interaction of constituent gravitons among themselves and with the external particles. Therefore, it is most natural to consider the process which would also be possible if we were to replace the coherent background states by number eigenstates. As we consider the decay of a particle, this leads to $\Delta N=N^{\prime}-N=-1$.

Moreover, this choice of $\Delta N$ conserves the expectation value of the energy, as it should. Since the coupling of the coherent state and the produced particles vanishes asymptotically, the only relevant contributions to the Hamiltonian come from the free massive gravitons, $\hat{H}_{0}^{(\Phi)}$, and from the free external particles, $\hat{H}_{0}^{(\Psi)}$ :

$$
\left\langle N\left|\hat{H}_{0}^{(\Phi)}\right| N\right\rangle=\left\langle N^{\prime}\left|\hat{H}_{0}^{(\Phi)}\right| N^{\prime}\right\rangle+\left\langle 0\left|\hat{b}_{\vec{p}} \hat{b}_{\vec{p}^{\prime}} \hat{H}_{0}^{(\Psi)} \hat{b}_{\vec{p}}^{\dagger} \hat{b}_{\vec{p}^{\prime}}^{\dagger}\right| 0\right\rangle .
$$

This simple argument in terms of the free Hamiltonians only works, however, when gravity couples to an external field, as we assumed in our calculation. If scattering happens due to self-coupling, then the interaction term gives a non-zero contribution to the energy even asymptotically because of graviton interaction in the coherent state. Only if one could calculate this contribution, it would be possible to argue in terms of the energy expectation value. In any case, the precise value of $\Delta N$ does not matter for our conclusions.

\subsection{Calculation of the rate}

Using $\Delta N \ll N$, we obtain the matrix element:

$$
\left\langle N^{\prime}|\hat{\Phi}| N\right\rangle=\frac{1}{\sqrt{2 m V}}\left(e^{i m t} \sqrt{N^{\prime}}+e^{-i m t} \sqrt{N}\right)\left(1-\frac{\Delta N^{2}}{8 N}\right),
$$

where the $1 / N$-correction comes from the overlap of different coherent states. It vanishes in the semi-classical limit $\Delta N=0$. The $S$-matrix element subsequently becomes:

$$
\begin{aligned}
\mathscr{A}= & \frac{(2 \pi)^{4}}{\sqrt{2 m V}} \mathscr{K}\left(-p, p^{\prime}\right) \boldsymbol{\delta}^{(3)}\left(\vec{p}+\vec{p}^{\prime}\right)\left(1-\frac{\Delta N^{2}}{8 N}\right) \\
& \left(\delta\left(p_{0}+p_{0}^{\prime}-m\right) \sqrt{N^{\prime}}+\delta\left(p_{0}+p_{0}^{\prime}+m\right) \sqrt{N}\right) .
\end{aligned}
$$

The amplitude consists of two parts. The first one describes a process where a graviton leaves the bound state and the second one corresponds to adding a graviton to the bound state. The second process cannot occur because of energy conservation so that the term will be dropped. 
As is derived in detail in [1], the rate of particle production is

$$
\Gamma=\frac{2 \sqrt{\frac{m^{2}}{4}-m_{\Psi}^{2}} N}{M_{p}^{2} m^{2}}\left(\frac{m^{2}}{2}+m_{\Psi}^{2}\right)^{2}\left(1-\frac{\Delta N^{2}-4 \Delta N}{4 N}\right) .
$$

We observe that particle production to first order only takes place for light particles, $m_{\Psi}<\frac{m}{2}$, as we expect it. In order to simplify the discussion, we specialize to massless external particles $\left(m_{\Psi}=0\right)$ :

$$
\Gamma=\frac{N m^{3}}{4 M_{p}^{2}}\left(1-\frac{\Delta N^{2}-4 \Delta N}{4 N}\right)=\frac{\Lambda^{2} V}{32 \pi}\left(1-\frac{\Delta N^{2}-4 \Delta N}{4 N}\right) .
$$

For dimensional reasons, this result does not come as a surprise. The rate must be proportional to the volume $V$. Since particle production can also be derived in the semi-classical treatment, i.e., when only $\hat{\Psi}$ is quantized but gravity is treated classically, the Planck mass $M_{p}$ should not appear so that we can only use $\Lambda$ to obtain the correct mass dimension. In one Hubble volume $V \propto \Lambda^{-1.5}$, the rate matches the result of [2].

Before we proceed to our main result, the quantum break-time, we want to briefly check to what extent our approach is consistent with the semi-classical result. To this end, we estimate the power of produced particles. In the semi-classical treatment, the Hubble horizon radiates like a black body of temperature $T \sim \sqrt{\Lambda}$. According to the Stefan-Boltzman law, this yields the emitted power $P \sim T^{4} A$, where $A$ is the area of the horizon. Since $A \sim \Lambda^{-1}$, we get the semi-classical power

$$
P_{\mathrm{s}-\mathrm{c}} \sim \Lambda
$$

As a pair of produced particles has the energy $\sqrt{\Lambda}$, our quantum result for the decay rate (4.6) leads to the consistent result

$$
P_{\mathrm{q}} \sim \Lambda
$$

Restoring factors of $\hbar$ for a moment, $P_{\mathrm{q}} \sim \hbar \Lambda$, we note that particle production vanishes in the classical limit $\hbar \rightarrow 0$, as it should. That we are able to reproduce further properties of GibbonsHawking radiation, in particular the suppression of the production of high-energetic quanta, is discussed in [1].

\subsection{Quantum break-time}

In summary, we have found a crucial difference to the semi-classical treatment: In our quantum description of de Sitter, the back reaction of particle production on the space-time leads to a change of the coherent state $|N\rangle$ into a different $\left|N^{\prime}\right\rangle$, which is either a different coherent state or a decohered one. On the order of one background graviton leaves the coherent state each Hubble time, due to decay into free quanta or due to rescattering.

Even if hypothetically we were allowed to maintain only processes which preserve the coherence of the state of background gravitons, this would anyway inevitably lead us to a quantum evolution which has no counterpart in the semi-classical picture: Semi-classically, de Sitter is an eternal state with no clock. Therefore, even a "clean" transition between the two different coherent states $|N\rangle$ and $\left|N^{\prime}\right\rangle$ gives an intrinsically quantum evolution of the state which cannot be matched 
by anything in the classical theory. As we have seen, it is moreover impossible to maintain only processes which preserve the coherence of the state. In particular, the decay process which we calculated explicitly also leads to decoherence. This is true since even if we did not include the self-interaction of gravitons, the exchange by virtual $\hat{\Psi}$-particles will lead to their rescattering and subsequent decoherence. In other words, decay and rescattering go hand in hand due to the structure of spin-2 coupling.

With the help of the rate (4.6), it is easy to calculate the time-scale after which the back reaction to the de Sitter state becomes important. This happens when a macroscopic number of gravitons $\Delta N$ of the order of $N$ has decayed. At this point, the resulting quantum state $|N-\Delta N\rangle$ can no longer - even approximately - reproduce the initial de Sitter metric and the classical description stops being valid. Consequently, we obtain the quantum break-time

$$
t_{\mathrm{q}} \sim \Gamma^{-1} N \sim \frac{N}{\sqrt{\Lambda}}
$$

in agreement with $[2,4]$. Rewriting in terms of more conventional parameters, we get

$$
t_{\mathrm{q}} \sim \frac{M_{P}^{2}}{\Lambda^{\frac{3}{2}}} .
$$

We argue that $t_{\mathrm{q}}$ is physically meaningful even though $t_{\mathrm{q}}>t_{\mathrm{cl}}$. The reason for this is that $t_{\mathrm{cl}}$ could be increased by choosing a better classical approximation than (2.6), which takes into account classical nonlinear interactions. But for any choice of approximation, the decoherence mechanism will continue to work and lead to a significant deviation from the classical solution after $t_{\mathrm{q}}$. Thus, (4.11) is the quantum break-time of de Sitter.

We will briefly comment on the implications of the quantum break-time on the symmetry properties of de Sitter. Unlike the standard treatment, for us de Sitter is not a vacuum state, but rather a particular coherent state defined in the Fock space with Minkowski vacuum. Correspondingly, the symmetry of de Sitter metric is not a symmetry of the vacuum of the fundamental theory, but rather emerges as an effective symmetry of the expectation value over a particular coherent state, i.e., it is a symmetry of the mean field description. Once the classical description breaks down after $t_{\mathrm{q}}$, also the de Sitter symmetry disappears.

As a final remark, it is interesting to study the effect of the number of species on the de Sitter quantum break-time. If we assume in our simple model that the graviton is coupled to a large number of particle species $\hat{\Psi}_{j}, \quad j=1,2, \ldots, \mathscr{N}_{\mathrm{sp}}$, then the presence of more species opens up more channels for Gibbons-Hawking particle production so that the rate increases by a factor of $\mathscr{N}_{\text {sp }}$. Correspondingly, the quantum break-times becomes shorter so that equation (4.11) takes the form:

$$
t_{\mathrm{q}} \sim \frac{1}{\sqrt{\Lambda}}\left(\frac{1}{\mathscr{N}_{\mathrm{sp}}} \frac{M_{P}^{2}}{\Lambda}\right)
$$

\section{Implications for the cosmological constant}

As suggested in [4], the concept of quantum break-time effectively promotes the cosmological constant problem from an issue of naturalness into a question of consistency. For any given value of the cosmological constant, the quantum break-time puts a consistency constraint on the classically 
describable duration of the universe. The older it is and the more species it contains, the lower is the bound on cosmological constant. Any patch with a given value of the Hubble parameter $H=\sqrt{\Lambda}$ can be described classically at most during the time $t_{\mathrm{q}} \sim\left(H^{-1} / \mathscr{N}_{\mathrm{sp}}\right)\left(M_{P}^{2} / H^{2}\right)$, as stated in equation (4.12).

It is interesting to apply this constraint to our Universe where the observed value of the cosmological constant is $\sqrt{\Lambda}=10^{-42} \mathrm{GeV}$. Let us see how long it is possible to describe it classically. Currently, the phenomenologically acceptable number of hidden sector species is bounded by $\mathscr{N}_{\mathrm{sp}} \sim 10^{32}$ because a larger number of species would lower the gravity cutoff below the TeVscale, which is excluded by current collider data [8]. Assuming this number, the observed value of the cosmological term would saturate the quantum break-time bound if our Universe were approximately $10^{100}$ years old. This age should not be confused with the Hubble time or the Hubble radius. It pertains to the entire duration of the classically describable history of our patch.

We can also apply the constraint in the other direction: Knowing the classical age of a given universe and the number of species, we can deduce an upper bound on $\Lambda$. For the present age $t_{\mathrm{u}}$ of our Universe, we obtain $H_{\max } \sim\left(M_{p}^{2} / t_{\mathrm{u}}\right)^{1 / 3}=10^{-1} \mathrm{GeV}$, where we set $\mathscr{N}_{\mathrm{sp}}=1$ to obtain a more robust bound. This means that the energy density associated to the cosmological constant, $\rho \propto$ $H^{2} M p^{2}$, can be at most $10^{-40}$ of the Planckian energy density. ${ }^{4}$ On the one hand, we cannot explain why the cosmological constant is as small as it is. On the other hand, however, the cosmological constant has to be small for consistency.

\section{Conclusion}

Following [2], we have first attempted to understand de Sitter space as mean field description of an underlying quantum theory. In a simplified but fully consistent quantum model of spin-2, we managed to reproduce the de Sitter metric for short enough time-scales. It is the expectation value of a coherent multi-graviton state of characteristic frequency given by the de Sitter Hubble parameter $\sqrt{\Lambda}$ and mean occupation number $N=M_{P}^{2} / \Lambda$.

Next, we took into account the coupling of the coherent state gravitons to external probe particles $\hat{\Psi}$. When the graviton field is treated classically, the quantum evolution of $\hat{\Psi}$-particles reproduces all the usual features of fields quantized on top of a fixed classical de Sitter background metric. We studied Gibbons-Hawking particle production as an example.

The main novelty in our quantum framework is that we are able to capture back reaction effects, which are invisible in the semi-classical treatment. Namely, we have shown that GibbonsHawking particle production and other quantum processes necessarily change an initial quantum coherent state. This back reaction inevitably leads to decoherence and as a result, the classical metric description of de Sitter stops being valid after a finite time. This is the quantum break-time given by (4.11).

As already pointed out in [4], the existence of a de Sitter quantum break-time may promote the cosmological constant problem from an issue of naturalness into a question of quantum consistency. The quantum break-time tells us that the older the classical universe is and the more particle species

\footnotetext{
${ }^{4}$ Including $N_{\mathrm{sp}} \sim 10^{32}$ would lead to $H_{\mathrm{max}} \sim 10^{-12} \mathrm{GeV}$ and an energy density of at most $10^{-62}$ of the Planckian value.
} 
it houses, the lower is the upper bound on its vacuum energy. ${ }^{5}$ For example, assuming the maximal number of phenomenologically acceptable species, the observed value of the cosmological constant would saturate the consistency bound if our Universe were $10^{100}$ years old.

By no means do we pretend to have understood the complete quantum picture of de Sitter space in full nonlinear Einstein gravity. However, the model we work with, despite being simple, does not only take us surprisingly far in reproducing the known properties of de Sitter space in a fundamentally new quantum language, but also naturally incorporates back reaction effects on the gravitational background. Of course, this may very well be simply a remarkable coincidence, but can also be an indication that we are on the right track in understanding the quantum nature of de Sitter space.

\section{Acknowledgements}

The work of G.D. was supported by the Humboldt Foundation under Alexander von Humboldt Professorship, the ERC Advanced Grant "Selfcompletion" (Grant No. 339169), FPA 2009-07908, CPAN (CSD2007-00042), HEPHACOSP-ESP00346, and by TR 33 "The Dark Universe". The work of C.G. was supported in part by the Humboldt Foundation and by Grants: FPA 2009-07908, CPAN (CSD2007-00042), and by the ERC Advanced Grant 339169 "Selfcompletion".

\section{References}

[1] G. Dvali, C. Gomez, and S. Zell, Quantum Break-Time of de Sitter, arXiv: 1701.08776 [hep-th].

[2] G. Dvali and C. Gomez, Quantum Compositeness of Gravity: Black Holes, AdS and Inflation, J. Cosmol. Astropart. Phys. 1401 (2014), 023, arXiv:1312.4795 [hep-th] .

[3] G. Dvali, D. Flassig, C. Gomez, A. Pritzel, and N. Wintergerst, Scrambling in the Black Hole Portrait, Phys. Rev. D88 (2013), 124041, arXiv: 1307.3458 [hep-th] .

[4] G. Dvali and C. Gomez, Quantum Exclusion of Positive Cosmological Constant?, Annalen Phys. 528 (2016), 68, arXiv:1412.8077 [hep-th].

[5] G. Dvali, S. Hofmann, and J. Khoury, Degravitation of the cosmological constant and graviton width, Phys. Rev. D76 (2007), 084006, arXiv: hep-th/ 0703027 .

[6] G. Dvali, Predictive power of strong coupling in theories with large distance modified gravity, New J. Phys. 8 (2006), 326, arXiv: hep-th/ 0610013.

[7] G. W. Gibbons and S. W. Hawking, Cosmological event horizons, thermodynamics, and particle creation, Phy. Rev. D15 (1977), 2738.

[8] G. Dvali, Black Holes and Large N Species Solution to the Hierarchy Problem, Fortschr. Phys. 58 (2010), 528, arXiv:0706.2050 [hep-th].

G. Dvali and M. Redi, Black Hole Bound on the Number of Species and Quantum Gravity at LHC, Phys. Rev. D77 (2008), 045027, arXiv:0710.4344 [hep-th].

G. Dvali and C. Gomez, Quantum Information and Gravity Cutoff in Theories with Species, Phys. Lett. B674 (2009), 303, arXiv:0812.1940 [hep-th].

\footnotetext{
${ }^{5}$ The age of the Universe is counted throughout the classical history of a given patch and should not be confused with the Hubble radius. Otherwise, the statement would be trivial.
} 